Isotropy theorem for arbitrary-spin cosmological fields

This content has been downloaded from IOPscience. Please scroll down to see the full text. JCAP03(2014)042

(http://iopscience.iop.org/1475-7516/2014/03/042)

View the table of contents for this issue, or go to the journal homepage for more

Download details:

IP Address: 147.96.14.16

This content was downloaded on 05/05/2014 at $14: 34$

Please note that terms and conditions apply. 


\title{
Isotropy theorem for arbitrary-spin cosmological fields
}

\author{
J.A.R. Cembranos, A.L. Maroto and S.J. Núñez Jareño \\ Departamento de Física Teórica, Universidad Complutense de Madrid, \\ Avenida Complutense s/n, Madrid, 28040 Spain \\ E-mail: cembra@ucm.es, maroto@ucm.es, sjnjareno@ucm.es
}

Received January 29, 2014

Revised February 25, 2014

Accepted March 7, 2014

Published March 21, 2014

\begin{abstract}
We show that the energy-momentum tensor of homogeneous fields of arbitrary spin in an expanding universe is always isotropic in average provided the fields remain bounded and evolve rapidly compared to the rate of expansion. An analytic expression for the average equation of state is obtained for Lagrangians with generic power-law kinetic and potential terms. As an example we consider the behavior of a spin-two field in the standard Fierz-Pauli theory of massive gravity. The results can be extended to general space-time geometries for locally inertial observers.
\end{abstract}

Keywords: particle physics - cosmology connection, dark matter theory, dark energy theory ArXiv ePrint: 1311.1402 


\section{Contents}

1 Introduction 1

2 Homogeneous fields and virial theorem 3

3 A spin-2 example $\quad 5$

4 General geometrical backgrounds and discussion 6

\section{Introduction}

One of the main limitations on the use of vectors or higher-spin fields in cosmology is the high degree of isotropy of the universe on large scales [1]. A homogeneous field of non-zero spin generically breaks isotropy by selecting preferred directions in space.

However in recent years there has been a growing interest in the possibility of using vectors fields (abelian or non-abelian) as dark matter [2], dark energy [3-7] or inflaton candidates [8-25]. In these cases the anisotropy problem is avoided thanks to the use of particular field configurations (temporal components, triads, etc [26-33]) that guarantee an isotropic energy-momentum tensor. Also a more general result has been proved which shows that in the case of bounded fields which evolve rapidly as compared to the rate of expansion, the temporal average of the energy-momentum tensor is always isotropic for any field configuration. This means, that even anisotropic field configurations such as a linearly polarized field would give rise in average to an isotropic energy-momentum tensor. This result was obtained by using a generalization of virial theorem and applies both to abelian [34] and non-abelian [35] theories, with arbitrary potentials and with or without gauge-fixing terms.

The generality of this result for homogeneous vectors suggests that the isotropy property could be a general feature of any field theory for arbitrary spin with the only requirements of large scale homogeneity, boundedness and rapid evolution. In this work we prove that this is indeed the case and present a general isotropy theorem for arbitrary-spin cosmological fields.

Unlike previous works in which explicit Lagrangian densities were used, in the case of generic theories as those we will consider in this work, the explicit dependence of the Lagrangian on the metric tensor is not fixed a priori. This means that we cannot use the Hilbert form of the energy-momentum tensor:

$$
T^{\mu \nu}=-\frac{2}{\sqrt{g}} \frac{\delta S}{\delta g_{\mu \nu}},
$$

as our starting point. In order to avoid this difficulty, we will make use of the so called Belinfante-Rosenfeld [36, 37] energy-momentum tensor, which allows to relate the Hilbert energy-momentum with the canonical one by means of the use of some extra terms. Unexpectedly, we will show how this relation between the canonical and Hilbert forms is intimately related to the anisotropy issue.

For clarification, let us thus start by briefly reviewing the standard Belinfante-Rosenfeld approach in Minkowski space-time [38] and consider a Lagrangian density depending only on the fields (labelled by $A$ ) and their gradients:

$$
\mathcal{L} \equiv \mathcal{L}\left[\phi^{A}, \partial_{\mu} \phi^{A}\right] .
$$


Under an infinitesimal $x$-dependent translation $x^{\mu} \rightarrow x^{\mu}+\delta a^{\mu}(x)$, the field and its gradient change as [38]:

$$
\begin{aligned}
\delta \phi^{A} & =\delta a^{\mu}(x) \partial_{\mu} \phi^{A}(x), \\
\delta \partial_{\mu} \phi^{A}(x) & =\delta a^{\nu}(x) \partial_{\nu} \partial_{\mu} \phi^{A}(x)+\partial_{\mu}\left[\delta a^{\nu}(x)\right] \partial_{\nu} \phi^{A}(x) .
\end{aligned}
$$

By imposing:

$$
0=\delta \int d^{4} x \mathcal{L}=-\int d^{4} x \delta a_{\nu} \partial_{\mu} \Theta^{\mu \nu}
$$

we obtain that the canonical energy-momentum tensor, defined as

$$
\Theta^{\mu \nu}=-\eta^{\mu \nu} \mathcal{L}+\frac{\partial \mathcal{L}}{\partial\left(\partial_{\mu} \phi^{A}\right)} \partial^{\nu} \phi^{A}
$$

is conserved:

$$
\partial_{\mu} \Theta^{\mu \nu}=0
$$

This tensor is nothing but the Noether current associated to the symmetry under space-time translations. Notice that although it is conserved, $\Theta_{\mu \nu}$ is not necessarily symmetric.

However, this current is not unique, and we can add a new piece:

$$
\partial_{\rho} \tilde{\Theta}^{\rho \mu \nu}
$$

with $\tilde{\Theta}^{\rho \mu \nu}$ antisymmetric in the first two indices. This new piece does not modify the value of the Noether charge because it is a total derivative, neither its time conservation because of its antisymmetry,

$$
\begin{aligned}
Q^{\nu} & =\int d^{3} x\left(\Theta^{0 \nu}+\partial_{\rho} \tilde{\Theta}^{\rho 0 \nu}\right) \\
& =\int d^{3} x\left(\Theta^{0 \nu}+\partial_{i} \tilde{\Theta}^{i 0 \nu}\right)=\int d^{3} x \Theta^{0 \nu} ; \\
\frac{d Q^{\nu}}{d t} & =\int d^{3} x\left(\partial_{\mu} \Theta^{\mu \nu}+\partial_{\mu} \partial_{\rho} \tilde{\Theta}^{\rho \mu \nu}\right)=0 .
\end{aligned}
$$

We are interested in a symmetric energy-momentum tensor, i.e. that required to appear on the right hand side of Einstein equations. The new piece that must be added read $[39,40]$ :

$$
T^{\mu \nu}=\Theta^{\mu \nu}-\frac{1}{2} \partial_{\rho}\left(S^{\rho \mu \nu}+S^{\mu \nu \rho}-S^{\nu \rho \mu}\right)
$$

with

$$
S^{\mu \nu \rho}=\Pi_{A}^{\mu} \Sigma^{\nu \rho} \phi^{A},
$$

where $\Sigma^{\nu \rho}$ are the antisymmetric Lorentz group generators in the corresponding representation and

$$
\Pi_{A}^{\mu}=\frac{\partial \mathcal{L}}{\partial\left(\partial_{\mu} \phi^{A}\right)},
$$


is the generalized momentum associated to $\phi^{A}$. $T^{\mu \nu}$ is the symmetric Belinfante-Rosenfeld energy-momentum tensor which agrees with the Hilbert energy-momentum tensor obtained from variations with respect to the metric (1.1) as shown in [36, 37, 41].

Both, the canonical energy-momentum tensor $\Theta^{\mu \nu}$ and the Belifante-Rosenfeld tensor $T^{\mu \nu}$ can be written in a curved space-time in a straightforward way by using minimal coupling, simply changing ordinary derivatives by covariant ones, i.e. we will work with:

$$
\begin{aligned}
T^{\mu \nu} & =\Theta^{\mu \nu}+\nabla_{\rho} \tilde{\Theta}^{\rho \mu \nu} \\
& =\Theta^{\mu \nu}-\frac{1}{2} \nabla_{\rho}\left(S^{\rho \mu \nu}+S^{\mu \nu \rho}-S^{\nu \rho \mu}\right) .
\end{aligned}
$$

Notice, that the form of the Lagrangian guarantees that only first derivatives of the fields will appear in $\Theta_{\mu \nu}$.

\section{Homogeneous fields and virial theorem}

Following [34] and [35], we can use a generalization of the virial theorem in order to obtain interesting results for the average energy-momentum tensor of homogeneous fields $\phi^{A}(t)$. Before writing the most general theorem, let us consider a Friedmann-Lemaître-RobertsonWalker (FLRW) metric for simplification:

$$
d s^{2}=d t^{2}-a(t) d \vec{x}^{2} .
$$

With these assumptions, the $\tilde{\Theta}^{\rho \mu \nu}$ tensor is also homogeneous.

Our aim is taking the temporal average of the energy momentum tensor during periods $\mathcal{T} \ll H^{-1}$, where $H$ is the Hubble parameter $H=\dot{a} / a$. Particularly, we are interested in the average value of $\nabla_{\rho} \tilde{\Theta}^{\rho \mu \nu}$ as this term will be the cause of the anisotropies.

$$
\left\langle\nabla_{\rho} \tilde{\Theta}^{\rho \mu \nu}\right\rangle=\frac{1}{\mathcal{T}} \int_{t}^{t+\mathcal{T}} d t^{\prime}\left(\nabla_{\rho} \tilde{\Theta}^{\rho \mu \nu}\right)\left(t^{\prime}\right)
$$

with

$$
\nabla_{\rho} \tilde{\Theta}^{\rho \mu \nu}=\partial_{0} \tilde{\Theta}^{0 \mu \nu}+\left(\Gamma_{\delta \rho}^{\rho} \tilde{\Theta}^{\delta \mu \nu}+\Gamma_{\delta \rho}^{\mu} \tilde{\Theta}^{\rho \delta \nu}+\Gamma_{\delta \rho}^{\nu} \tilde{\Theta}^{\rho \mu \delta}\right) .
$$

We can neglect the term in brackets on the right hand side of the equation (2.3) if the temporal derivative is larger than the expansion rate, i.e. $\partial_{0} \tilde{\Theta} \gg H \tilde{\Theta}$. If the system oscillates with an effective period $\tau, \partial_{0} \Theta \sim \tau^{-1} \Theta$, then the condition for neglecting that term will be:

$$
\tau^{-1} \gg \mathcal{T}^{-1} \gg H
$$

In this limit the energy-momentum tensor expressed in components reads

$$
\begin{aligned}
T^{00} & =\Pi_{A}^{0} \partial_{0} \phi^{A}-\mathcal{L}-\frac{1}{2} \partial_{0}\left(S^{000}\right) \\
& =\Pi_{A}^{0} \partial_{0} \phi^{A}-\mathcal{L} ; \\
T^{0 j} & =-\frac{1}{2} \partial_{0}\left(S^{00 j}+S^{0 j 0}-S^{j 00}\right)=0 ; \\
T^{j j} & =-g^{j j} \mathcal{L}-\frac{1}{2} \partial_{0}\left(S^{0 j j}+S^{j j 0}-S^{j 0 j}\right) \\
& =-g^{j j} \mathcal{L}-\partial_{0}\left(\Pi_{A}^{j} \Sigma^{j 0} \phi^{A}\right) ; \\
T^{j k} & =-\frac{1}{2} \partial_{0}\left(\Pi_{A}^{0} \Sigma^{j k} \phi^{A}+\Pi_{A}^{j} \Sigma^{k 0} \phi^{A}+\Pi_{A}^{k} \Sigma^{0 j} \phi^{A}\right),
\end{aligned}
$$


with $k \neq j$. The antisymmetry of the Lorentz group generators, $\Sigma^{\mu \nu}$, has been used for simplification.

On the other hand, (2.2) becomes

$$
\begin{aligned}
\left\langle\nabla_{\rho} \tilde{\Theta}^{\rho \mu \nu}\right\rangle & =\frac{1}{\mathcal{T}} \int_{t}^{t+\mathcal{T}} d t^{\prime} \partial_{0} \tilde{\Theta}^{0 \mu \nu}\left(t^{\prime}\right) \\
& =\frac{\tilde{\Theta}^{0 \mu \nu}(t+\mathcal{T})-\tilde{\Theta}^{0 \mu \nu}(t)}{\mathcal{T}} .
\end{aligned}
$$

As can be seen from (2.9), if the field evolution is periodic or bounded, the right-hand side vanishes as compared to $\left\langle T^{00}\right\rangle$ for sufficiently large $\mathcal{T}$. In fact, the ratio can be estimated as $\left\langle\nabla_{\rho} \tilde{\Theta}^{\rho \mu \nu}\right\rangle /\left\langle T^{00}\right\rangle \sim \mathcal{O}(\tau / \mathcal{T})$. That leads us to the following average energy-momentum tensor:

$$
\begin{aligned}
& \left\langle T^{00}\right\rangle=\left\langle\Pi_{A}^{0} \partial_{0} \phi^{A}-\mathcal{L}\right\rangle ; \\
& \left\langle T^{0 j}\right\rangle=T^{0 j}=0 ; \\
& \left\langle T^{j j}\right\rangle=\left\langle-g^{j j} \mathcal{L}\right\rangle ; \\
& \left\langle T^{j k}\right\rangle=0 ; k \neq j,
\end{aligned}
$$

which is explicitly isotropic. Notice that as commented before, the anisotropies in the exact (non-averaged) tensor indeed come from the new terms that must be added in the BelinfanteRosenfeld approach in order to get the symmetric expression.

Moreover, using these results we can also express the average equation of state in this suggestive form:

$$
\omega=\frac{\langle p\rangle}{\langle\rho\rangle}=\frac{\langle\mathcal{L}\rangle}{\left\langle\Pi_{A}^{0} \partial_{0} \phi^{A}-\mathcal{L}\right\rangle}=\frac{\langle\mathcal{L}\rangle}{\langle\mathcal{H}\rangle},
$$

with $\mathcal{H}$ the Hamiltonian of the system.

There are other ways of writing this quantity:

$$
\omega=\frac{\left\langle\Pi_{A}^{0} \partial_{0} \phi^{A}\right\rangle}{\langle\mathcal{H}\rangle}-1
$$

Or by using the equation $\partial_{0} \phi^{A}=\frac{\partial \mathcal{H}}{\partial \Pi_{A}^{0}}$ :

$$
\omega=\frac{\left\langle\Pi_{A}^{0} \frac{\partial \mathcal{H}}{\partial \Pi_{A}^{0}}\right\rangle}{\langle\mathcal{H}\rangle}-1 .
$$

Another form is reached by using the Euler-Lagrange equation for $\phi^{A}, \nabla_{\mu} \Pi_{A}^{\mu}=\frac{\partial \mathcal{L}}{\partial \phi^{A}}$ :

$$
\omega=\frac{\left\langle\partial_{0}\left(\Pi_{A}^{0} \phi^{A}\right)-\partial_{0} \Pi_{A}^{0} \phi^{A}\right\rangle}{\langle\mathcal{H}\rangle}-1=\frac{\left\langle-\frac{\partial \mathcal{L}}{\partial \phi^{A}} \phi^{A}\right\rangle}{\langle\mathcal{H}\rangle}-1,
$$

where we have also applied the extension of the virial theorem to $\Pi_{A}^{0} \phi^{A}$, i.e. $\left\langle\partial_{0}\left(\Pi_{A}^{0} \phi^{A}\right)\right\rangle=0$.

From (2.16) and (2.17), it can be seen that the following average equation is satisfied

$$
\left\langle\Pi_{A}^{0} \frac{\partial \mathcal{H}}{\partial \Pi_{A}^{0}}+\frac{\partial \mathcal{L}}{\partial \phi^{A}} \phi^{A}\right\rangle=0
$$


The last equation results very helpful when considering theories where the kinetic and potential terms add separately as simple power-laws in the following form

$$
\mathcal{H}=\left(\lambda^{A B} g_{00} \Pi_{A}^{0} \Pi_{B}^{0}\right)^{n_{T}}+\left(M_{A B} \phi^{A} \phi^{B}\right)^{n_{V}},
$$

where $\lambda^{A B}$ and $M_{A B}$ are constant matrices. In such a case, equation (2.18) relates $T$ and $V$ in the following form

$$
\langle T\rangle=\frac{n_{V}}{n_{T}}\langle V\rangle .
$$

By using (2.17), we can obtain an analytic expression for $\omega$ independent of initial conditions or particular polarization of $\phi^{A}$ :

$$
\omega=\frac{2 n_{V}\langle V\rangle}{\langle T+V\rangle}-1=\frac{2 n_{V}}{1+\frac{n_{V}}{n_{T}}}-1
$$

Notice that this result is also independent of the field spin. For instance, for the usual case with $n_{T}=1$, the behaviour of the equation of state is the same as that for scalar [42] or vector $[34,35]$ fields:

$$
\omega=\frac{n_{V}-1}{n_{V}+1}
$$

Note that fast oscillating fields can have associated a negative effective equation of state parameter. In this sense, they are potential new models of dark energy or inflation. Indeed, we have shown that this result does not depend on the spin. Similar approaches for scalar fields have been already considered in the literature [43-45]. Another potential interest of these results comes from the possibility of avoiding the anisotropy typically expected during the reheating period in inflationary models based on vectors or higher-spin fields.

\section{$3 \quad$ A spin-2 example}

As an example, we will apply the previous results to the Fierz-Pauli theory of massive gravity on a curved space-time background given by the Lagrangian ${ }^{1}$

$$
\begin{aligned}
\mathcal{L}= & \frac{M_{\mathrm{Pl}}^{2}}{8}\left[\nabla_{\alpha} h^{\mu \nu} \nabla^{\alpha} h_{\mu \nu}-2 \nabla_{\alpha} h_{\mu}^{\alpha} \nabla_{\beta} h^{\mu \beta}+2 \nabla_{\alpha} h_{\mu}^{\alpha} \nabla^{\mu} h_{\beta}^{\beta}-\nabla_{\alpha} h_{\mu}^{\mu} \nabla^{\alpha} h_{\nu}^{\nu}\right. \\
& \left.-m_{g}^{2}\left(h_{\mu \nu} h^{\mu \nu}-\left(h_{\mu}^{\mu}\right)^{2}\right)\right] .
\end{aligned}
$$

The momentum of this field can be written as

$$
\Pi_{\mu \nu}^{0}=\frac{\partial \mathcal{L}}{\partial\left(\nabla_{0} h^{\mu \nu}\right)}=\frac{M_{\mathrm{Pl}}^{2}}{4}\left[\nabla^{0} h_{\mu \nu}-2 \delta_{(\mu}^{0} \nabla_{\alpha} h_{\nu)}^{\alpha}+\delta_{(\mu}^{0} \nabla_{\nu)} h_{\alpha}^{\alpha}+g_{\mu \nu} \nabla_{\alpha} h^{\alpha 0}-g_{\mu \nu} \nabla^{0} h_{\alpha}^{\alpha}\right],
$$

where $A^{(\mu} B^{\nu)}=\left(A^{\mu} B^{\nu}+A^{\nu} B^{\mu}\right) / 2$.

\footnotetext{
${ }^{1}$ Note that we are assuming a minimal gravitational coupling for the spin-2 field. There are more general options [46] but they are not relevant for the isotropy theorem presented in this analysis.
} 
Imposing homogeneity, considering a FLRW metric and exploiting the fact that $h_{\mu \nu}$ is symmetric, the momenta and the Lagrangian take the form

$$
\begin{aligned}
\Pi_{0 \mu}^{0} & =0 \\
\Pi_{i j}^{0} & =\frac{M_{\mathrm{Pl}}^{2}}{4} \partial^{0} h_{i j}, \quad i \neq j \\
\Pi_{i i}^{0} & =-\frac{M_{\mathrm{Pl}}^{2}}{4} \sum_{j \neq i} \partial^{0} h_{j j} ; \\
\mathcal{L} & =\frac{M_{\mathrm{Pl}}^{2}}{8}\left[\partial_{0} h_{i j} \partial^{0} h^{i j}-\partial_{0} h_{i}^{i} \partial^{0} h_{j}^{j}-m_{g}^{2}\left(h_{\mu \nu} h^{\mu \nu}-\left(h_{\mu}^{\mu}\right)^{2}\right)\right],
\end{aligned}
$$

where we have neglected the expansion rate with respect to the temporal variation of the field. We will also need the explicit expression for the Hamiltonian. Under the same assumptions, we can write

$$
\mathcal{H} \equiv \Pi_{\mu \nu}^{0} \partial_{0} h^{\mu \nu}-\mathcal{L}=\frac{M_{\mathrm{Pl}}^{2}}{8}\left[\partial_{0} h_{i j} \partial^{0} h^{i j} \partial_{0} h_{i}^{i} \partial^{0} h_{j}^{j}+m_{g}^{2}\left(h_{\mu \nu} h^{\mu \nu}-\left(h_{\mu}^{\mu}\right)^{2}\right)\right] .
$$

As it can be seen, the Lagrangian and the Hamiltonian take the classical structure $\mathcal{L}=T-V$ and $\mathcal{H}=T+V$. If the field evolves under the conditions for applying the virial theorem, then (2.18) holds. Consequently,

$$
\left\langle\Pi_{\mu \nu}^{0} \frac{\partial \mathcal{H}}{\partial \Pi_{\mu \nu}^{0}}+\frac{\partial \mathcal{L}}{\partial h^{\mu \nu}} h^{\mu \nu}\right\rangle=\left\langle\Pi_{\mu \nu}^{0} \nabla_{0} h^{\mu \nu}+\frac{\partial \mathcal{L}}{\partial h^{\mu \nu}} h^{\mu \nu}\right\rangle=\langle 2 T-2 V\rangle=0,
$$

where one of the Hamilton equations has been used in the first equality. We can conclude that the behaviour of the field will be that of non-relativistic matter by using the last average equation and (2.14):

$$
\omega=\frac{\langle\mathcal{L}\rangle}{\langle\mathcal{H}\rangle}=\frac{\langle T-V\rangle}{\langle T+V\rangle}=0 .
$$

Therefore, given the weak coupling to matter fields, a homogeneous spin-two massive graviton can contribute to the dark matter density. The massive graviton has been already studied as a dark matter candidate by assuming an isotropic stochastic background [47, 48]. However, even an anisotropic coherent evolution could be taken into account as a viable model since, as shown before, it does not introduce an important amount of anisotropy in the background geometry.

\section{General geometrical backgrounds and discussion}

Finally, let us extend this result to a more general space-time geometry by considering an inertial observer located at $x_{0}^{\mu}=0$ and write the metric around it using Riemann normal coordinates:

$$
g_{\mu \nu}(x)=\eta_{\mu \nu}+\frac{1}{3} R_{\mu \alpha \nu \beta} x^{\alpha} x^{\beta}+\ldots
$$

If the following conditions hold:

1. The Lagrangian depends only on the fields and their gradients. 
2. The field evolves rapidly:

$$
\left|R_{\lambda \mu \nu}^{\gamma}\right| \ll\left(\omega_{A}\right)^{2}, \quad \text { and } \quad\left|\partial_{j} S^{\mu \nu \rho}\right| \ll\left|\partial_{0} S^{\mu \nu \rho}\right|, \quad \text { for } \quad j=1,2,3 ;
$$

for any component of the Riemann tensor. $\omega_{A}$ is the characteristic frequency of $\phi^{A}$.

3. $S^{\mu \nu \rho}$, i.e. $\phi^{A}$ and $\Pi_{A}^{0}$, remains bounded in the evolution.

then, the second condition implies that if the averaging times satisfy

$$
\left|R_{\lambda \mu \nu}^{\gamma}\right| \ll \mathcal{T}^{-2} \ll\left(\omega_{A}\right)^{2},
$$

we are in a normal neighborhood and we can neglect the second term in (4.1) so that we can work locally in a Minkowskian space-time. In the normal neighborhood of the observer, $\tilde{\Theta}^{\rho \mu \nu}$ can also be considered as a homogeneous field. In such a region, it is then possible to rewrite all the above equations in Minkowski space-time $(a(t)=1)$. Accordingly, it is possible to neglect the right-hand side in (2.9) and prove that the mean value of the energy-momentum tensor is isotropic. Thus, if oscillations are fast compared to the curvature scale, the average energy-momentum tensor takes the perfect fluid form for any locally inertial observer.

\section{Acknowledgments}

This work has been supported by MICINN (Spain) project numbers FIS2011-23000, FPA2011-27853-01 and Consolider-Ingenio MULTIDARK CSD2009-00064.

\section{References}

[1] Planck collaboration, P.A.R. Ade et al., Planck 2013 results. XXIII. Isotropy and statistics of the $C M B$, arXiv:1303.5083 [INSPIRE].

[2] A.E. Nelson and J. Scholtz, Dark Light, Dark Matter and the Misalignment Mechanism, Phys. Rev. D 84 (2011) 103501 [arXiv:1105.2812] [INSPIRE].

[3] C. Armendariz-Picon, Could dark energy be vector-like?, JCAP 07 (2004) 007 [astro-ph/0405267] [INSPIRE].

[4] C.G. Boehmer and T. Harko, Dark energy as a massive vector field, Eur. Phys. J. C 50 (2007) 423 [gr-qc/0701029] [INSPIRE].

[5] J. Beltran Jimenez and A.L. Maroto, A cosmic vector for dark energy, Phys. Rev. D 78 (2008) 063005 [arXiv:0801.1486] [INSPIRE].

[6] J. Beltran Jimenez and A.L. Maroto, Cosmological electromagnetic fields and dark energy, JCAP 03 (2009) 016 [arXiv:0811.0566] [INSPIRE].

[7] J. Beltran Jimenez and A.L. Maroto, The electromagnetic dark sector, Phys. Lett. B 686 (2010) 175 [arXiv:0903.4672] [INSPIRE].

[8] L.H. Ford, Inflation Driven By A Vector Field, Phys. Rev. D 40 (1989) 967 [InSPIRE].

[9] K. Dimopoulos, Can a vector field be responsible for the curvature perturbation in the Universe?, Phys. Rev. D 74 (2006) 083502 [hep-ph/0607229] [INSPIRE].

[10] A. Golovnev, V. Mukhanov and V. Vanchurin, Vector Inflation, JCAP 06 (2008) 009 [arXiv:0802.2068] [INSPIRE].

[11] T. Koivisto and D.F. Mota, Vector Field Models of Inflation and Dark Energy, JCAP 08 (2008) 021 [arXiv:0805.4229] [INSPIRE]. 
[12] K. Bamba, S.-'i. Nojiri and S.D. Odintsov, Inflationary cosmology and the late-time accelerated expansion of the universe in non-minimal Yang-Mills- $F(R)$ gravity and non-minimal vector-F $(R)$ gravity, Phys. Rev. D 77 (2008) 123532 [arXiv:0803.3384] [INSPIRE].

[13] A. Maleknejad, M.M. Sheikh-Jabbari and J. Soda, Gauge Fields and Inflation, Phys. Rept. 528 (2013) 161 [arXiv:1212.2921] [INSPIRE].

[14] K. Yamamoto, M.-a. Watanabe and J. Soda, Inflation with Multi-Vector-Hair: The Fate of Anisotropy, Class. Quant. Grav. 29 (2012) 145008 [arXiv:1201.5309] [INSPIRE].

[15] M.-a. Watanabe, S. Kanno and J. Soda, Inflationary Universe with Anisotropic Hair, Phys. Rev. Lett. 102 (2009) 191302 [arXiv:0902.2833] [INSPIRE].

[16] B. Himmetoglu, C.R. Contaldi and M. Peloso, Instability of anisotropic cosmological solutions supported by vector fields, Phys. Rev. Lett. 102 (2009) 111301 [arXiv:0809.2779] [InSPIRE].

[17] A.E. Gumrukcuoglu, B. Himmetoglu and M. Peloso, Scalar-Scalar, Scalar-Tensor and Tensor-Tensor Correlators from Anisotropic Inflation, Phys. Rev. D 81 (2010) 063528 [arXiv: 1001.4088] [INSPIRE].

[18] K. Murata and J. Soda, Anisotropic Inflation with Non-Abelian Gauge Kinetic Function, JCAP 06 (2011) 037 [arXiv:1103.6164] [INSPIRE].

[19] A. Maleknejad and M.M. Sheikh-Jabbari, Revisiting Cosmic No-Hair Theorem for Inflationary Settings, Phys. Rev. D 85 (2012) 123508 [arXiv:1203.0219] [InSPIRE].

[20] A. Maleknejad and M.M. Sheikh-Jabbari, Gauge-flation: Inflation From Non-Abelian Gauge Fields, Phys. Lett. B 723 (2013) 224 [arXiv: 1102.1513] [INSPIRE].

[21] A. Maleknejad and M.M. Sheikh-Jabbari, Non-Abelian Gauge Field Inflation, Phys. Rev. D 84 (2011) 043515 [arXiv:1102.1932] [InSPIRE].

[22] P. Adshead and M. Wyman, Chromo-Natural Inflation: Natural inflation on a steep potential with classical non-Abelian gauge fields, Phys. Rev. Lett. 108 (2012) 261302 [arXiv:1202.2366] [INSPIRE].

[23] P. Adshead and M. Wyman, Gauge-flation trajectories in Chromo-Natural Inflation, Phys. Rev. D 86 (2012) 043530 [arXiv: 1203.2264] [InSPIRE].

[24] K. Yamamoto, Primordial Fluctuations from Inflation with a Triad of Background Gauge Fields, Phys. Rev. D 85 (2012) 123504 [arXiv:1203.1071] [INSPIRE].

[25] M.M. Sheikh-Jabbari, Gauge-flation Vs Chromo-Natural Inflation, Phys. Lett. B 717 (2012) 6 [arXiv:1203.2265] [INSPIRE].

[26] J. Cervero and L. Jacobs, Classical Yang-Mills Fields in a Robertson-walker Universe, Phys. Lett. B 78 (1978) 427 [INSPIRE].

[27] M. Henneaux, Remarks on spacetime symmetries and nonabelian gauge fields, J. Math. Phys. 23 (1982) 830 [InSPIRE].

[28] Y. Hosotani, Exact Solution to the Einstein-Yang-Mills Equation, Phys. Lett. B 147 (1984) 44 [INSPIRE].

[29] D.V. Gal'tsov and M.S. Volkov, Yang-Mills cosmology: Cold matter for a hot universe, Phys. Lett. B 256 (1991) 17 [INSPIRE].

[30] D.V. Gal'tsov, Non-Abelian condensates as alternative for dark energy, arXiv:0901.0115 [INSPIRE].

[31] Y. Zhang, Inflation with quantum Yang-Mills condensate, Phys. Lett. B 340 (1994) 18 [INSPIRE].

[32] Y. Zhang, An Exact solution of a quark field coupled with a Yang-Mills field in de Sitter space, Class. Quant. Grav. 13 (1996) 2145 [INSPIRE]. 
[33] E. Elizalde, A.J. Lopez-Revelles, S.D. Odintsov and S.Y. Vernov, Cosmological models with Yang-Mills fields, Phys. Atom. Nucl. 76 (2013) 996 [arXiv:1201.4302] [INSPIRE].

[34] J.A.R. Cembranos, C. Hallabrin, A.L. Maroto and S.J. Núñez Jareño, Isotropy theorem for cosmological vector fields, Phys. Rev. D 86 (2012) 021301 [arXiv:1203.6221] [INSPIRE].

[35] J.A.R. Cembranos, A.L. Maroto and S.J. Núñez Jareño, Isotropy theorem for cosmological Yang-Mills theories, Phys. Rev. D 87 (2013) 043523 [arXiv:1212.3201] [INSPIRE].

[36] F.J. Belinfante, On the current and the density of the electric charge, the energy, the linear momentum and the angular momentum of arbitrary fields, Physica 7 (1940) 449.

[37] L. Rosenfeld, Sur le tenseur d'impulsion-énergie, Mem. Acad. Roy. Belg. Sci. 18 (1940) 1.

[38] C. Itzykson and J.-B. Zuber, Quantum field theory, McGraw-Hill (1980).

[39] W. Greiner and J. Reinhardt, Field Quantization, Springer (1996).

[40] A. Dobado, A. Gómez-Nicola, A.L. Maroto and J.R. Peláez, Effective Lagrangians for the Standard Model, Springer (1997).

[41] S.V. Babak and L.P. Grishchuk, The Energy momentum tensor for the gravitational field, Phys. Rev. D 61 (2000) 024038 [gr-qc/9907027] [InSPIRE].

[42] M.S. Turner, Coherent scalar-field oscillations in an expanding universe, Phys. Rev. D 28 (1983) 6 [INSPIRE].

[43] T. Damour and V.F. Mukhanov, Inflation without slow roll, Phys. Rev. Lett. 80 (1998) 3440 [gr-qc/9712061] [INSPIRE].

[44] A.R. Liddle and A. Mazumdar, Inflation during oscillations of the inflaton, Phys. Rev. D 58 (1998) 083508 [astro-ph/9806127] [INSPIRE].

[45] T.S. Koivisto and N.J. Nunes, Inflation and dark energy from three-forms, Phys. Rev. D 80 (2009) 103509 [arXiv:0908.0920] [INSPIRE].

[46] F. Berkhahn, S. Hofmann, F. Kühnel, P. Moyassari and D. Dietrich, Island of Stability for Consistent Deformations of Einstein's Gravity, Phys. Rev. Lett. 108 (2012) 131102 [arXiv:1106.3566] [INSPIRE].

[47] S.L. Dubovsky, P.G. Tinyakov and I.I. Tkachev, Massive graviton as a testable cold dark matter candidate, Phys. Rev. Lett. 94 (2005) 181102 [hep-th/0411158] [INSPIRE].

[48] M. Pshirkov, A. Tuntsov and K.A. Postnov, Constraints on the massive graviton dark matter from pulsar timing and precision astrometry, Phys. Rev. Lett. 101 (2008) 261101 [arXiv:0805.1519] [INSPIRE]. 